Check for updates

Cite this: Chem. Sci., 2019, 10, 6341

๑ All publication charges for this article have been paid for by the Royal Society of Chemistry

Received 13th February 2019

Accepted 8th May 2019

DOI: $10.1039 / c 9 s c 00749 k$

rsc.li/chemical-science

\title{
Molecular mechanism of polyketide shortening in anthraquinone biosynthesis of Photorhabdus luminescens $\dagger$
}

\author{
Qiuqin Zhou, ${ }^{a}$ Alois Bräuer, ${ }^{b}$ Hélène Adihou, ${ }^{a}$ Maximilian Schmalhofer, (DD ${ }^{b}$ \\ Patricia Saura, (D) ${ }^{\text {b }}$ Gina L. C. Grammbitter, ${ }^{a}$ Ville R. I. Kaila, (DD ${ }^{\mathrm{b}}$ Michael Groll (D)*b \\ and Helge B. Bode (iD *a
}

\begin{abstract}
Anthraquinones, a widely distributed class of aromatic natural products, are produced by a type II polyketide synthase system in the Gram-negative bacterium Photorhabdus luminescens. Heterologous expression of the antABCDEFGHI anthraquinone biosynthetic gene cluster in Escherichia coli identified Antl as an unusual lyase, catalysing terminal polyketide shortening prior to formation of the third aromatic ring. Functional in vitro and in vivo analysis of Antl using X-ray crystallography, structure-based mutagenesis, and molecular simulations revealed that Antl converts a defined octaketide to the tricyclic anthraquinone ring via retroClaisen and Dieckmann reactions. Thus, Antl catalyses a so far unobserved multistep reaction in this PKS system.
\end{abstract}

\section{Introduction}

Polyketide natural products attract significant attention due to their remarkable biological and pharmacological activities. Their structural diversity derives from only a few building blocks assembled by different polyketide synthase systems (PKS): ${ }^{1,2}$ type I PKS represent elaborate multifunctional enzyme complexes responsible for the synthesis of macrolides including erythromycin and candicin, ${ }^{3}$ whereas type III PKS catalyse the formation of stilbenes, chalcones, and pyrones. ${ }^{4} \mathrm{On}$ the other hand, type II PKS constitute discrete proteins that act in an iterative manner to synthesise polycyclic aromatic compounds such as anthraquinones (AQs), tetracyclines and doxorubicins. ${ }^{5,6}$ First, a specific acyl carrier protein (ACP) is loaded with an $\alpha$-carboxylated precursor, and the activated unit is then transferred onto the corresponding ketosynthase. Next, iterative elongation cycles with malonyl-coenzyme A building blocks form the octaketide framework. ${ }^{1}$ Subsequently, ketoreductases, cyclases and aromatases convert the unstable and highly reactive octaketide into the corresponding target compounds. Notably, the variability of the natural products can be further increased by tailoring enzymes, which are not part of

${ }^{a}$ Molekulare Biotechnologie, Fachbereich Biowissenschaften, Buchmann Institute for Molecular Life Sciences (BMLS), Goethe Universität Frankfurt, Max-von-Laue-Str. 15, Max-von-Laue-Str. 9, 60438 Frankfurt am Main, Germany. E-mail: h.bode@bio. uni-frankfurt.de

${ }^{b}$ Center for Integrated Protein Science Munich (CIPSM), Department of Chemistry, Technische Universität München, Lichtenbergstraße 4, 85748 Garching, Germany. E-mail: michael.groll@tum.de

$\dagger$ Electronic supplementary information (ESI) available. See DOI: $10.1039 / \mathrm{c} 9 \mathrm{sc} 00749 \mathrm{k}$ the gene cluster and therefore can be difficult to identify. Yet, these catalysts are specific and perform only minor modifications such as alkylations, hydroxylations, or oxidations, while preserving the octaketide skeleton.

Type I and type III PKS subclasses are well-known from bacteria, fungi, and plants. ${ }^{1}$ In contrast, type II PKS have only been characterised in Gram-positive Streptomyces and other actinomycetes. $^{5}$ We identified a biosynthetic gene cluster encoding a type II PKS in the Gram-negative entomopathogenic bacterium Photorhabdus luminescens, an enzyme system that enables the biosynthesis of AQs. ${ }^{7}$ Notably, along with the antimicrobial aurachins $s^{8}$ and pyxidicyclines ${ }^{9}$ from myxobacteria, AQs represent another fascinating example of the type II PKS machinery. Here, we have introduced the anthraquinone gene cluster antABCDEFGHI from P. luminescens in E. coli. Surprisingly, the formed AQ-256 product exhibits a heptaketide framework, which is unusual since polyphenolic polyketides derived from bacterial type II PKS systems are generally octaketides. ${ }^{1}$ Our present study reveals that AntI shortens the octaketide intermediate 1 to the final AQ heptaketide. Although this type of reaction is uncommon, a related mechanism has been described for Ayg1 $\mathrm{p}^{10}$ from Aspergillus fumigatus and WdYg1 $1 \mathrm{p}^{11}$ from Wangiella dermatitidis. Both fungal proteins are part of PKS systems involved in the biosynthesis of melanin pigments.

We provide insights into the molecular mechanism of AntI via biochemical, crystallographic, computational, and functional characterisations, and show how this enzyme catalyses an elimination reaction, followed by cyclisation to the anthraquinone ring. Taken together, our combined findings elucidate an interesting type of polyketide shortening mechanism. 


\section{Results and discussion}

The gene cluster encoding the anthraquinone biosynthetic machinery (antABCDEFGHI) in P. luminescens was heterologously expressed in $E$. coli (strain $\mathrm{EC}^{\mathrm{AQ}}$ ). This approach allowed detection of the central metabolite 1,3,7-trihydroxyanthracene9,10-dione (AQ-256), a tricyclic aromatic compound not found in wild-type $E$. coli (Fig. 1). Yet, methylated derivatives such as AQ-270a (one methyl group) and AQ-284a (two methyl groups) were identified in the natural producer $P$. luminescens (Fig. 2). As these molecules were not detected in our engineered $\mathrm{EC}^{\mathrm{AQ}}$ strain, this suggests that the responsible methyltransferases are absent in the ant cluster, and therefore must be located elsewhere in the Photorhabdus genome. Notably, the $\mathrm{EC}^{\mathrm{AQ}}$-system is accompanied by several shunt products (SP) such as $\mathbf{S P 1}, \mathbf{S P}^{\mathbf{E C}} \mathbf{1}$ and $\mathbf{S P}^{\mathbf{E C}_{2}}$ (Fig. 2). One reason for this observation might be that the expression level of the individual genes in the heterologous host is not balanced. Nevertheless, all shunt products could be traced back to the common precursor molecule 1 harboring an octaketide skeleton as its basic structural element, which is covalently bound to the acyl carrier protein AntF via a phosphopantetheine (PPT) prosthetic group (see below). To elucidate the formation of these distinct compounds, we selectively removed individual genes from our $\mathrm{EC}^{\mathrm{AQ}}$ operon. We focused on classical type II PKS enzymes such as the ketoreductase AntA (C9 carbonyl reduction), aromatase AntH (first cyclisation, C7/ C12), and cyclase AntC (second cyclisation, C5/C14). The resulting intermediates were identified by UV-VIS spectroscopy and mass spectrometry (ESI Fig. 1-3, Tables 1-2†). Mutants constructed in P. luminescens (ESI Fig. $4 \dagger$ ) and labelling experiments in E. coli (ESI Fig. $3 \dagger$ ) allowed assignment of the reaction cascade based on known biosynthetic pathways ${ }^{1,2}$ (ESI Fig. $5 \dagger$ ).

Most polyphenolic polyketides derived from bacterial type II PKS systems are octaketides. ${ }^{1}$ In this respect, AQ-256 with its heptaketide framework is exceptional. Moreover, the local alignment search in the NCBI databank revealed that AntI (Uniprot: Q7MZT8) is predominantly found in Photorhabdus species. Thus, AntI is a promising candidate for polyketide shortening, followed by a unique cyclisation of the third aromatic ring in AQ biosynthesis (Fig. 1). In order to confirm our hypothesis, we generated a deletion mutant of antI in P. luminescens ( $\mathrm{PL}^{\Delta \mathrm{antr}}$ ). As expected, the modified strain lost the ability to produce AQ-256 and its methylated derivatives (Fig. 2), and only the nitrogen-containing octaketide derivative SP1 was formed. Next, we removed antI from our engineered $E$. coli strain $\left(\mathrm{EC}^{\mathrm{AQ \Delta} \Delta \mathrm{antI}}\right)$ and analysed the resulting product pattern. As expected, AQ-256 is no longer present. However, in contrast to the Photorhabdus $\Delta$ antI mutant, which produced only the SP1 metabolite, we now observed several compounds. Via detailed characterisation of the $\mathrm{EC}^{\mathrm{AQ} \Delta \text { antI }}$ lysate, we identified SP1-5, $\mathbf{S P}^{\mathbf{E C}} \mathbf{1}$, and $\mathbf{S P}^{\mathbf{E C}}$, all of which could unambiguously be shown to derive from molecule 1 (Fig. 2, ESI Fig. $5 \dagger$ ). In summary, (i) the dihydropyridine moiety of SP1 results from 1 by assimilation of intracellular $\mathrm{NH}_{3}$; (ii) $\mathbf{S P 4}$ is formed from $\mathbf{S P 1}$ after dehydration and decarboxylation; (iii) utahmycin $\mathrm{A}^{12}$ (SP5) is an oxidation product of SP4; (iv) 3,8-dihydroxy-1-methyl-anthraquinone-2carboxylic acid (DMAC, $\mathbf{S} \mathbf{S P}^{\mathbf{E C}} \mathbf{1}$ ) is the oxidized product of an aldol-condensation of $\mathbf{1}$; and (v) aloesaponarin II $\left(\mathbf{S P}^{\mathbf{E C}} 2\right)$ results from the spontaneous decarboxylation of $\mathbf{S P}^{\mathbf{E C} \mathbf{1}}$. The assignment of these shunt products strongly indicates that AntI orchestrates the cyclisation of the third ring system in the AQ biosynthesis. In this reaction, 4-(3-acetyl-5-hydroxy-4-oxo-1,4-dihydronaphthalen2-yl)-3-oxobutanoic acid (1) bound to AntF via the PPT-prosthetic group possibly acts as the AntI substrate that is converted to 8hydroxy-1,10-dihydroanthracene-2,3,9(4H)-trione (2, Fig. 1). We thus propose that AntI catalyses the last step in this reaction cascade, whereas formation of the aromatic quinone moiety of AQ-256 occurs in an enzyme-independent manner by keto-enoltautomerism and spontaneous oxidation.

The rapid turnover of the precursor molecule $\mathbf{1}$ in combination with the resulting large variety of different shunt products achieved in our modified $E$. coli system, encouraged us to additionally validate octaketide intermediate $\mathbf{1}$ as the true AntI substrate. To this end, the biosynthesis of the well-known blue

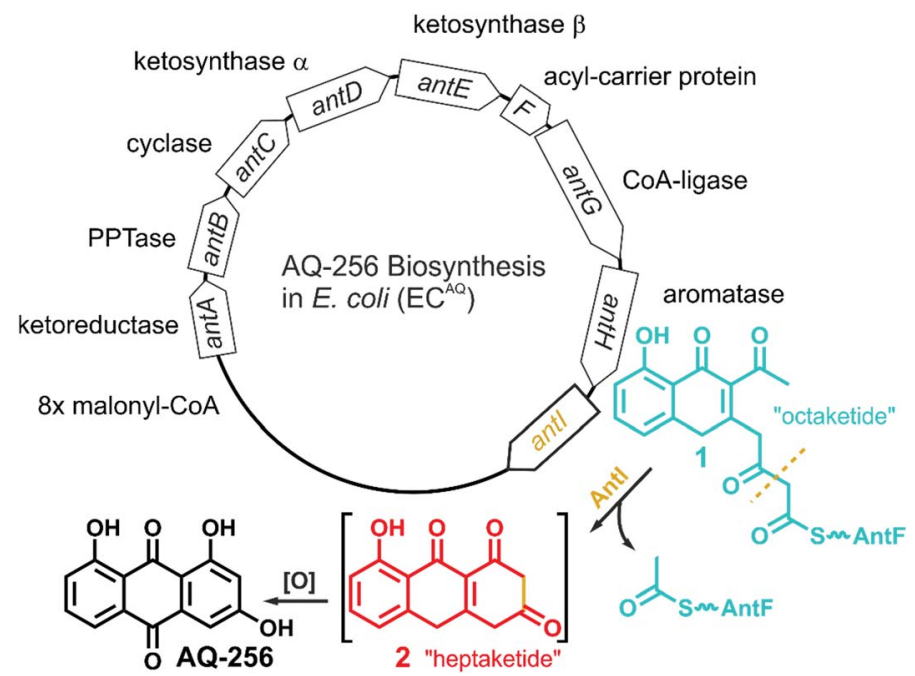

Fig. 1 Overview of the ant biosynthetic gene cluster from Photorhabdus luminescens. The AQ biosynthesis is highlighted with a focus on Antl catalyzed polyketide shortening. 

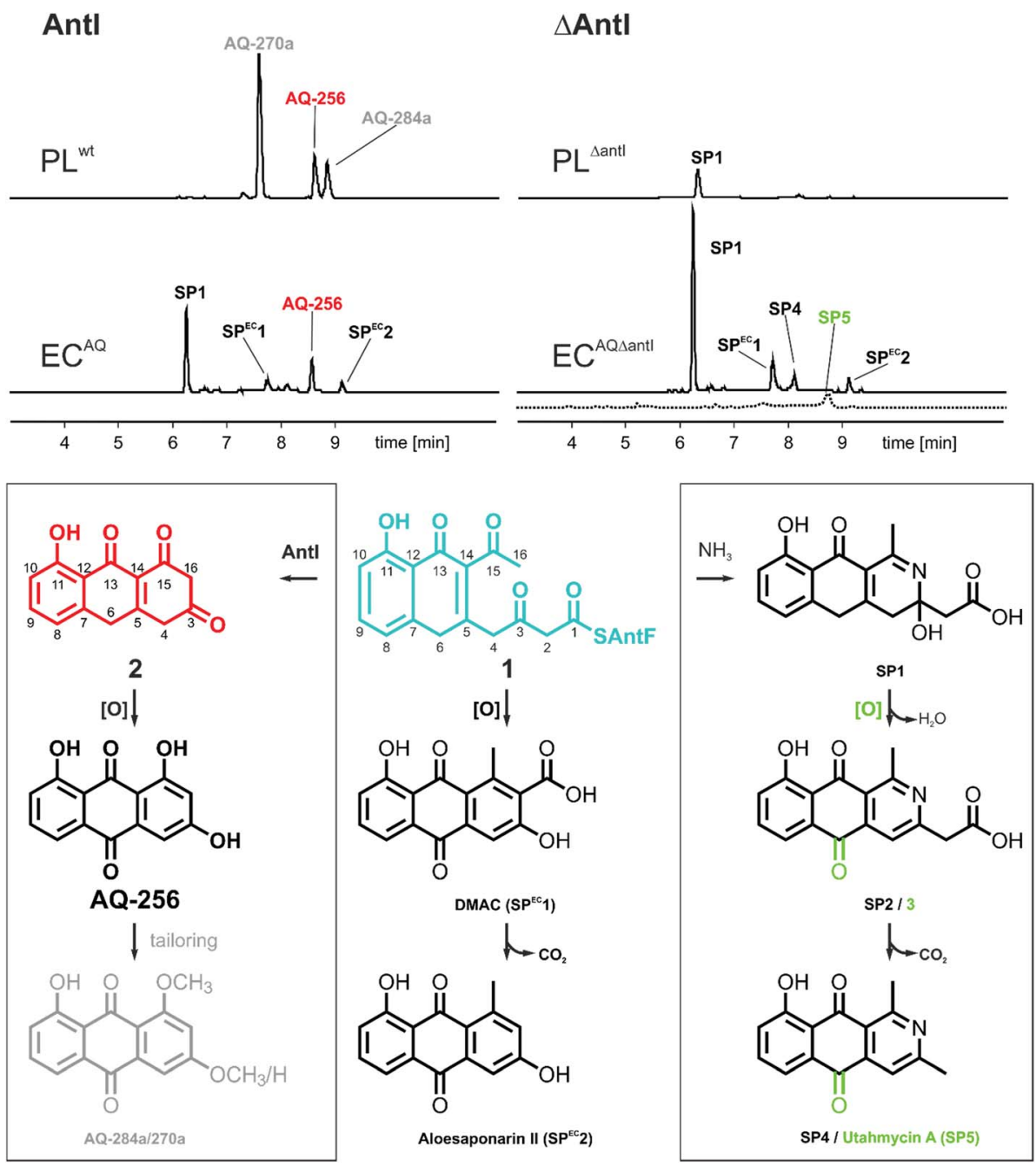

Fig. $2 \mathrm{HPLC/UV}$ (420 nm, solid line) and HPLC/MS (EIC m/z 254 for SP5, dashed line) analysis for polyketide production in the presence and absence of Antl in E. coli (EC) and Photorhabdus luminescens (PL). Identified key metabolites (top) and suggested biosynthesis pathways to these compounds (bottom) are shown.

pigment actinorhodin from Streptomyces coelicolor appeared to be ideal, as it transforms 1 into the octaketide 4-dihydro-9hydroxy-1-methyl-10-oxo-3-H-naphtho-[2,3-c]-pyran-3-(S)-acetate (S-DNPA). In this case, the stereospecific ketoreductase (Red1) acts as a tailoring enzyme by recognising the metabolite and reducing its $3 \beta$-keto group to a secondary alcohol. ${ }^{13}$ This peculiarity led us to extend the $\mathrm{EC}^{\mathrm{AQ} \Delta \mathrm{antI}}$ construct via a codonoptimized actVI ORF1 gene, encoding Red1, which resulted in the $\mathrm{EC}^{\mathrm{AQ} \Delta \text { antI+red1 }}$ strain. In this variant, $\mathbf{A Q - 2 5 6}$ was no longer produced, but we indeed detected the compound $\boldsymbol{S}$-DNPA (Fig. 3a), confirming the activity of Red1 in our model system. These results further prove that octaketide 1 is formed, and then subsequently shortened and cyclised by AntI.

With the identification of AntI's substrate (1), we wanted to obtain a deeper mechanistic insight into the catalytic properties of this fascinating enzyme. AntI was therefore heterologously expressed in $E$. coli, purified, and crystallised with one subunit in the asymmetric unit. Native diffraction data were collected to $1.85 \AA$ resolution, and the structure was phased by singlewavelength anomalous dispersion using selenomethionine labelling (PDB ID: 6HXA, $R_{\text {free }}=0.209$, ESI Table $3 \dagger$ ). The structure reveals that AntI functions as a homodimer, with each subunit comprising two domains (Fig. 4a) that are connected by a short linker sequence (residues 130-134). The N-terminal domain (residues 7-130) comprises an $\alpha$-helix bundle $(\alpha 1-\alpha 5)$ that orchestrates the arrangement of the two subunits in a backto-back orientation with a contact area of $2055 \AA^{2}$. The Cterminal domain (residues 134-381) contains a twisted eightstranded $\beta$-sheet $(\beta 1-\beta 8)$, which is flanked by $7 \alpha$-helices $(\alpha 6-$ $\alpha 12$ ) exhibiting an $\alpha / \beta$-hydrolase fold (Fig. $4 \mathrm{c}$ ). ${ }^{14}$

AntI forms a rod-shaped particle (Fig. 4b) with its N- and Cterminal domains enclosing a conspicuous intramolecular substrate binding cavity with approximate dimensions of $8 \times 8 \times$ $20 \AA^{3}$. A DALI search ${ }^{15}$ demonstrated similarity of AntI with 2,6dihydroxy-pseudo-oxynicotine hydrolase (DHPON; PDB ID: 2JBW, with a sequence identity of $23 \%, \mathrm{~N}$-domain: $Z$-score $=10.6$, RMSD $=2.5 \AA$; C-domain: $Z$-score $=30.5, \mathrm{RMSD}=1.9 \AA$ $),{ }^{16}$ a C-C bond cleaving $\alpha / \beta$-hydrolase from the Gram-positive bacterium Arthrobacter nicotinovorans that is involved in nicotine degradation (Fig. 4c). The structural comparison of AntI with DHPON 
a
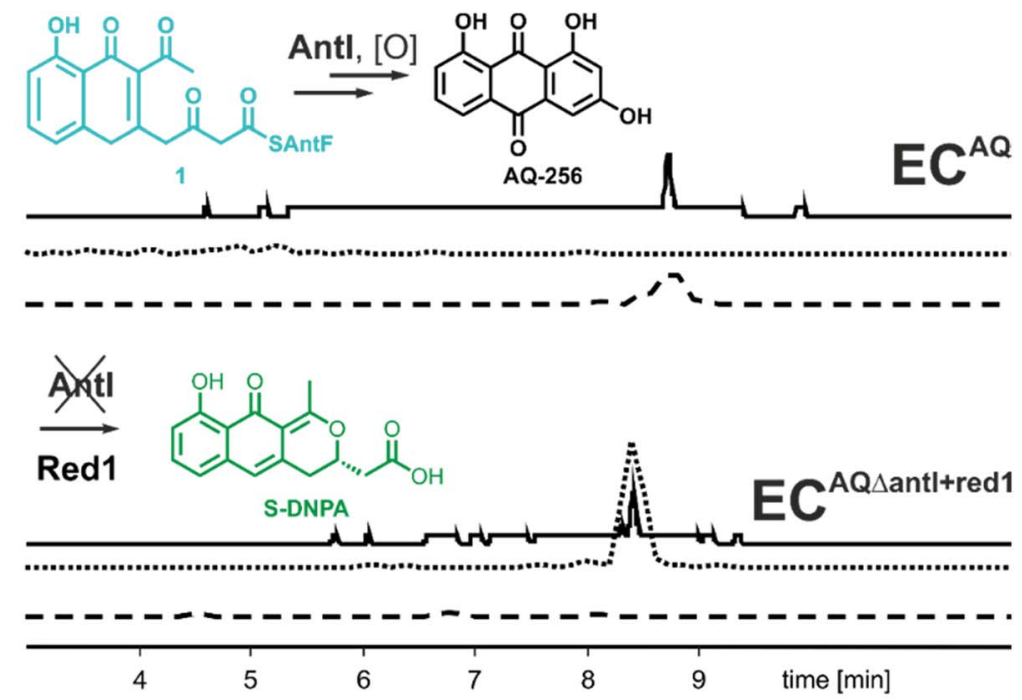

b

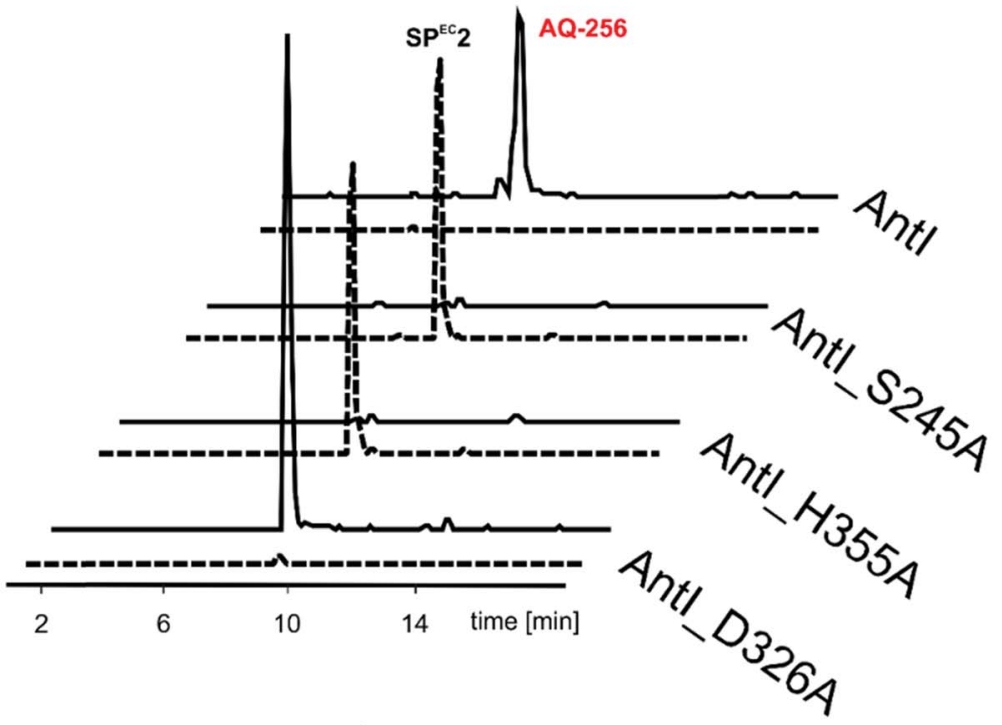

C

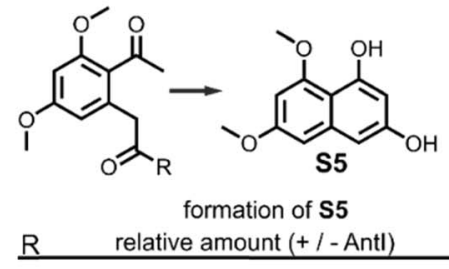

s1

$\mathrm{r}_{\mathrm{OH}}$

$-1-$

S2

ror

$100 / 100$

S3

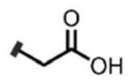

$-1-$

S4<smiles>COC(=O)C[12CH3]</smiles>

$3 /-$

Fig. 3 (a) HPLC analyses of E. coli expressing antABCDEFGHI and antABCDEFGH + actVI-ORF1 (encoding Red1). Besides UV traces (420 nm, solid line), EIC for AQ-256 (m/z $255[\mathrm{M}-\mathrm{H}]^{-}$, dashed line) and S-DNPA ( $\mathrm{m} / \mathrm{z} 287[\mathrm{M}+\mathrm{H}]^{+}$, dotted line) are shown. (b) In HPLC/MS analyses of $E$. coli expressing antABCDEFGH with the Antl-mutants S245A or H355A, metabolite 1 is no longer detected, but compound SPEC 2 ( $\mathrm{m} / z 253$ [M $\mathrm{H}^{-}$, dashed line) is formed. Mutation of D326 to alanine did not alter catalysis. (c) Conversion of model compounds S1-S4 into S5. 
a

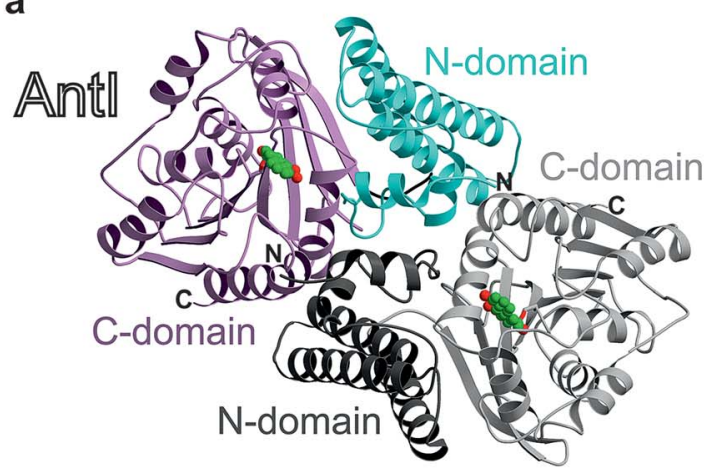

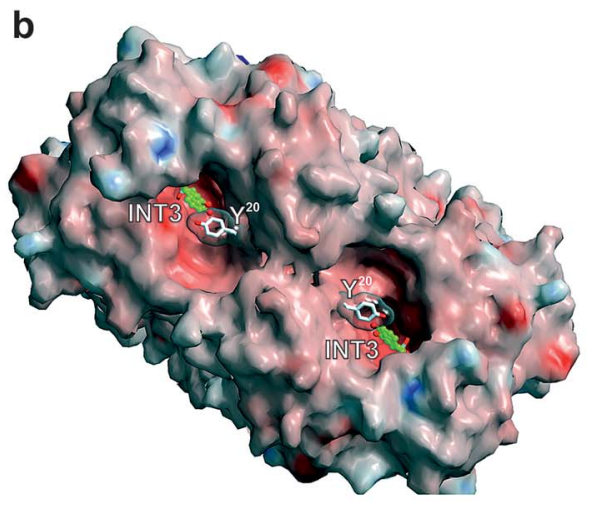

C
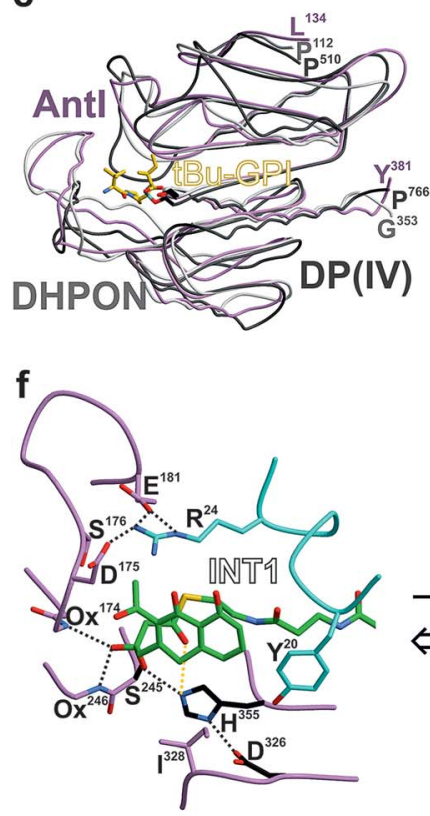

d

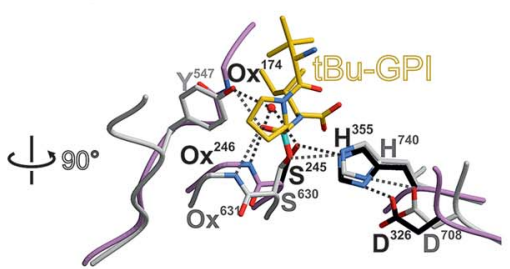

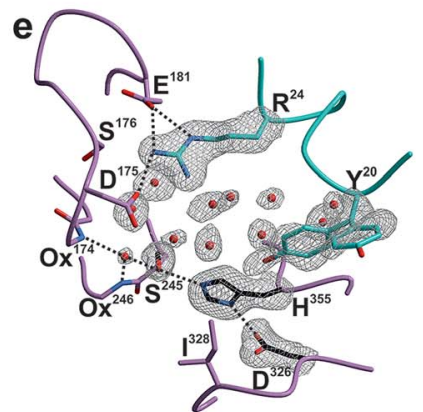
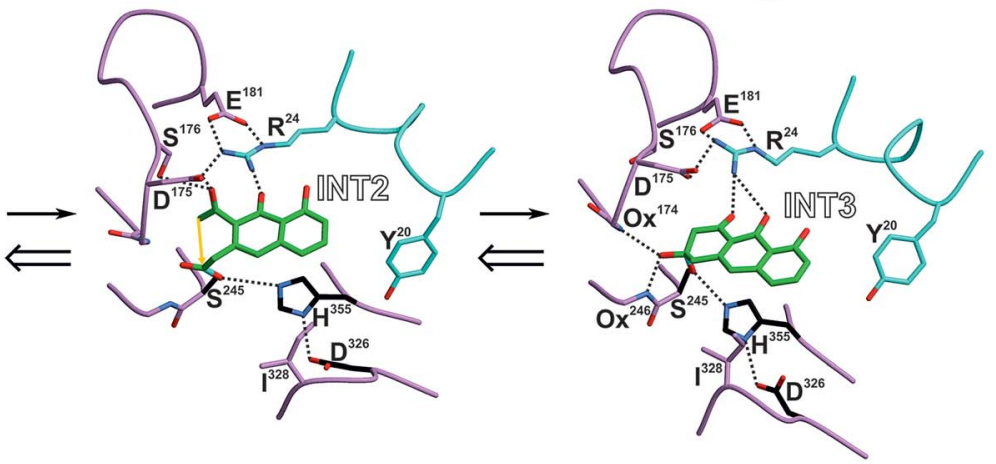

Fig. 4 Crystal structure of Antl. (a) The homodimer (ribbon plot) is depicted with a modelled intermediate (INT3, balls-and sticks, carbon atoms in green). $\mathrm{N}$ - and $\mathrm{C}$-domains of subunit A are coloured in pink and cyan, respectively; subunit B is shown in grey. (b) Surface representation of Antl. The active site of the lyase is solvent exposed. Colours highlight negative and positive electrostatic potentials contoured from $-40 k_{\mathrm{B}} T / \mathrm{C}_{\mathrm{C}}$ (red) to $+40 k_{\mathrm{B}} T / e$ (blue). (c) Superposition of the $\alpha / \beta$-hydrolase fold of Antl (residues 174-381, pink, PDB ID: 6HXA) with domains of 2,6dihydroxy-pseudo-oxynicotine hydrolase (DHPON, light grey, PDB ID: 2JBW) ${ }^{16}$ and the human dipeptidyl peptidase DP(IV)-tBu-GPI complex (grey, PDB ID: 2AJB). ${ }^{17}$ (d) Structural overlay of Antl with the active site of DP(IV) (C-atoms in grey) covalently bound to the $t$ Bu-GPI surrogate. Notably, the tetrahedral coordination fits into the active site of Antl, and identifies its putative oxyanion hole $\left(O_{x}\right)$, which is occupied by a water molecule. In Antl, the main chain atoms of Leu174NH and Phe246NH might form the oxyanion hole, while in DP(IV) Tyr631N and Tyr547OH take over this function. Hydrogen bonds are drawn as dashed lines. (e) Close up view of the active site of Antl showing the $2 F_{\mathrm{o}}-F_{\mathrm{c}}$ electron density map (grey meshes contoured to $1.0 \sigma$ ) for the catalytic triad, Tyr20 (alternative conformation which might function as the gate keeper) and Arg24. The abundance of well-defined water molecules (red spheres) is a clear indication for the possible substrate binding site. (f) Proposed multistep reaction sequence of Antl including a retro-Claisen reaction and a Dieckmann condensation. The INT1, INT2, and INT3 intermediates were modelled based on the DP(IV)-tBu-GPI complex. The ligand-bound Antl structures remains dynamically stable during the MD simulations (see ESI $\uparrow$ ). The calculations were performed in the reverse direction from INT3 to INT1.

reveals the catalytic centre of a serine-type protease composed of Ser245, Asp326, and His355. In the active site, Ser245 is positioned at the tip of a sharp turn between sheet $\beta 5$ and helix $\alpha 8$, called the nucleophilic elbow, ${ }^{\mathbf{1 4}}$ and points towards the helix dipole generated at the N-terminus of helix $\alpha 8$ (Fig. $4 \mathrm{~d}$ ). The mutation of Ser245 or His355 to alanine confirmed the catalytic importance of these residues (Fig. 3b). In contrast, the Asp326Ala mutant remains active. Thus, Asp326 polarises His355, but proton transfer from Ser245 to His355 is possible also without the aspartate residue. Inspection of the $2 F_{\mathrm{o}}-F_{\mathrm{c}}$-electron density map illustrates that the catalytic centre is enclosed by a cluster of bulk solvent molecules, the release of which might facilitate substrate binding (Fig. 4e). Consistent with the asymmetric hydrophilic rim of $\mathbf{1}$, which is composed of three oxygen atoms, the specificity pocket exhibits an amphiphilic character. The hydrophobic half of this pocket comprises Phe27, Ile244, Ile326, 
Val356, Leu358, and Ile361, whereas the polar part includes Arg24, Asp175, Ser176, Glu181, and Asp287. The central opening into the chamber is formed by Tyr20, Pro281, and Asp327. Interestingly, the sidechain of Tyr20 is observed in two alternative conformations (Fig. 4e), suggesting a regulatory role for this residue in allowing the substrate to access the active centre.

We aimed to obtain detailed insights into the reaction cascade of AntI. While the elimination reaction could clearly be assigned to AntI, the enzyme-dependency of the cyclisation resulting in the anthracene ring system still required experimental validation. We therefore synthesised surrogates $\mathbf{S 1}$ and S2 representing non-cyclised derivatives of the heptaketide intermediate (Fig. 3c, ESI Fig. 6†): S1 harbours a free carboxylate moiety, whereas the methoxy group of $\mathbf{S 2}$ mimics the sidechain of Ser245. Interestingly, S1 is stable in solution and in the presence of AntI, whereas $\mathbf{S 2}$ undergoes rapid ring closure to S5 even without AntI (Fig. 3c). These findings indicate that an oxoester with AntI has to be formed, to enable intermolecular cyclization. Hence, AntI is involved in the multistep biosynthetic route by catalyzing polyketide shortening and subsequent ring formation.

The next goal was to confirm the retro-Claisen reaction for shortening the octaketide skeleton to a heptaketide. In contrast to the Dieckmann condensation, this requires a strong nucleophile, which might represent the rate-limiting step in AntI catalysis. To this end, we determine whether AntI works like a tailoring enzyme that uses intermediate 1 as its substrate after hydrolysis from the PPT arm (containing a free carboxylate), or whether 1 is still covalently bound to its ACP AntF. We synthesised 2-carboxymethyl-benzoic acid (surrogate S3), as well as its corresponding methyl ester $\mathbf{S 4}$ which mimics metabolite $\mathbf{1}$. Both molecules remain stable in solution and in the presence of AntI. This observation is in contrast to the spontaneous enzymeindependent aromatisation of ester surrogate S2 (see above). Thus, compared to the Dieckmann condensation, we assume that AntF is mandatory for the retro-Claisen reaction.

To obtain mechanistic insights into the structure and dynamics of these reaction intermediates, we performed molecular simulations based on our experimentally solved structure of AntI. As a starting point for these computations, we used a surrogate pose of the hydrolytic domain of the homologous porcine dipeptidyl peptidase IV (DP(IV), residues 511-766, PDB ID: $2 \mathrm{AJB}, \mathrm{RMSD}$ of $145 \mathrm{C}^{\alpha}$-atoms $=2.7 \AA$, sequence identity $17 \%)$. DP(IV) was solved with the tripeptide tert-butyl-Gly-Pro-Ile ( $t \mathrm{Bu}-\mathrm{GPI})$, a surrogate that covalently binds in a $\mathrm{sp}^{3}$-configuration to the catalytic Ser630O ${ }^{\gamma}$ by forming an orthoester. ${ }^{17}$ Hereby, the alkoxide moiety of the inhibitor is negatively charged due to its strong interaction with the oxyanion hole. The tetrahedral coordination of $t \mathrm{Bu}-\mathrm{GPI}$ resembles the expected transition state structure with the alcoholate stabilised by hydrogen bonds to the backbone of Tyr631NH (3.1 $\AA$ ) and the sidechain of Tyr547OH (2.9 $\AA$ ). Intriguingly, superimposing the $\alpha / \beta$-hydrolase fold of DP(IV) with AntI reveals an excellent structural overlap of the catalytic residues (Fig. 4c). We observe that in AntI the putative oxyanion hole is occupied by a well-defined water molecule (Fig. 4d) that forms hydrogen-bonds to the backbone of Leu174NH (2.7 ̊) and Phe246NH (3.2 ̊).
In accordance with the structural similarities of the active site in AntI and DP(IV), we modelled the putative reaction intermediates INT1-INT3 and refined the protein-ligand complexes by classical molecular dynamics (MD) simulations. The procedure was initiated in reverse order, starting from a fully formed anthracene ring system at the same site as the tetrahedral hemiacetal carbon atom of $t \mathrm{Bu}-\mathrm{GPI}$ is bound. Thus, we first modelled the late product state INT3 which adopts a configuration having only one chiral centre out of plane with respect to the surrounding $\mathrm{sp}^{2}$-hybridised atoms. We find that INT3 remains stably positioned at the oxyanion hole, the catalytic centre, and Arg24 during our 100 ns MD simulations. Interestingly, Tyr20, which is located at the entry gate of the substrate binding-pocket, switches between an open and a closed conformation during the MD simulations (ESI Fig. $7 \dagger$ ). This finding is consistent with the two alternative positions of this residue in the X-ray structure (Fig. 4e). Furthermore, our simulations suggest that INT3 forms van der Waals contacts with Leu284 and Ile328, as well as hydrogen bonds between the biaryl hydroxyl and keto groups via Arg24. In addition, the aromatic ring system of INT3 is stabilised from both sides by cation- $\pi$ interactions with Arg24 and by $\pi$-stacking with His355 (Fig. 4f, left panel). These insights allowed us to analyse the Dieckmann condensation in more detail.

INT3 was used as the starting pose for modelling the INT2 state by following the enzymatic reaction cycle in reverse order. Thus, we opened the third ring of AQ-256 between C3 and C16, leading to an acetyl group and an ester bond at the nucleophile Ser245O ${ }^{\gamma}$ (INT2, Fig. 4f, central panel). The INT2 pose remains stable during the MD simulations, with less than 2 A fluctuation in the atomic positions of INT2 relative to INT3 (ESI Fig. $7 \dagger$ ). However, the INT2 ligand has an increased flexibility that leads to rotation of the acetyl group by $45^{\circ}$. In addition, the carbonyl oxygen coordinates to the sidechain of Ser176, forming close contacts with Asp175 that favour the enol tautomer of the ligand. In accordance with the AntI X-ray structure, the sidechain of Asp175 is in proximity to Asp287 (2.5 $⿱$ A) and, together with Glu181, forms an ion-pair with Arg24. This conformation facilitates deprotonation of the enol tautomer, via proton transfer to His355. Subsequently, the generated alkoxide might act as the nucleophile, initiating the Dieckmann condensation by intramolecular ring closure (Fig. 5a). The reaction takes place via a Bürgi-Dunitz angle in which the carbon atom in $\alpha$-position (C16) is perfectly oriented to form a covalent bond with the carbonyl group (C3) of the acyl-AntI complex leading to INT3 (Fig. 4f, right panel). Starting from INT2, this attack is regioselective from the $r$-face, while the backside is shielded from the catalytically active centre to exclude saponification that would result in shunt products.

We prolonged INT2 by an additional acetyl group, and converted the ester at the active site Ser245 back to $\mathrm{sp}^{3}$-hybridisation (C3) (INT1, Fig. 4f, left panel). In doing this, we expected that the flexible, ca. $20 \AA$ long PPT chain, introduces some structural uncertainty into the model. Intriguingly, the MD simulations of this state suggest that the oxyanion hole is rigid and dynamically stable over time, allowing us to properly refine the orientation of the introduced acyl-thioester moiety in INT1. 
a
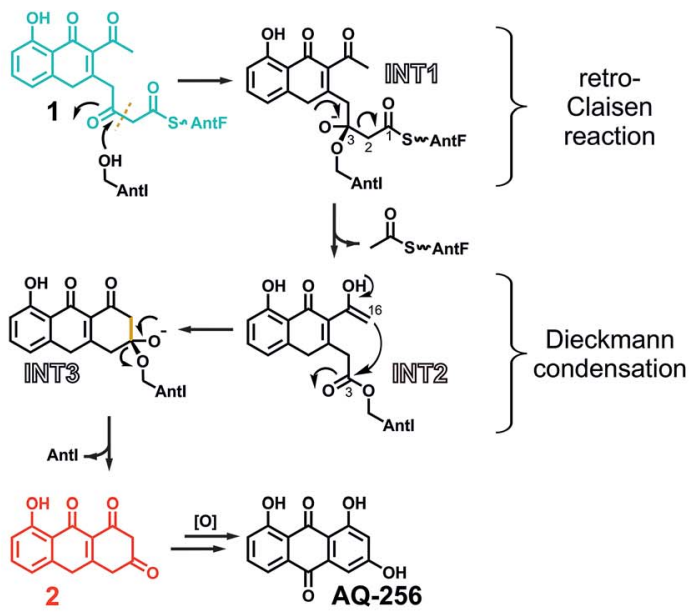

b

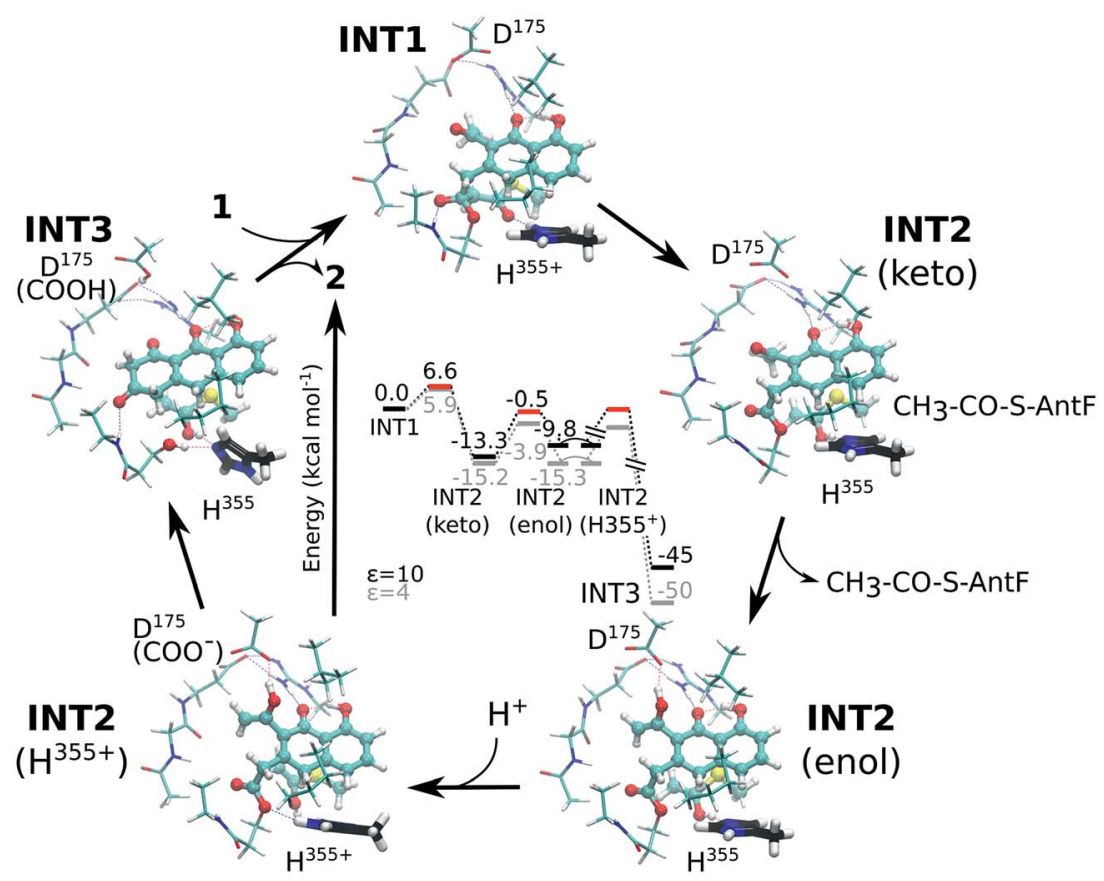

Fig. 5 (a) Proposed reaction scheme for the conversion of 1 into AQ-256 by Antl. (b) Quantum chemical DFT calculations (B3LYP-D3/def2$\mathrm{TZVP} / \varepsilon=4 / 10$ ) on a putative mechanism starting from INT1. See main text for detailed description.

The steric hindrance from the PPT arm and the adjacent acetyl group at C14 significantly increased in our calculations, relative to the INT2 and INT3 states. The predictions suggest that INT1 may re-orient towards the catalytically active serine residue in a new position induced by the tetrahedral configuration near the oxyanion hole (Fig. 4f, left panel). This conformation directs the carbonyl oxygen of the thioester at $\mathrm{C} 1$ towards His355, which might initiate the breakage of the adjacent $\mathrm{C} 2-\mathrm{C} 3$ bond. Due to the anionic charge distribution within the active site, both nitrogens of His355 might be protonated in the late state of INT2. In order to probe the energetics of these putative INT1INT3 intermediates, we performed quantum chemical density functional theory (DFT) calculations, starting from an active site model system constructed from an MD-relaxed snapshot of INT1 (Fig. 5b). The DFT calculations illustrate that the proton transfer from His355 to the carbonyl oxygen of the thioester group has a barrier of less than $10 \mathrm{kcal} \mathrm{mol}^{-1}$, and is exergonic by 13-15 $\mathrm{kcal} \mathrm{mol}^{-1}$, indicating that the reaction is both kinetically and thermodynamically feasible. Hereby hydrolysis of the ester at the nucleophilic Ser245 is supressed, and the subsequent Dieckmann condensation favoured. For the ketoenol tautomerisation forming INT2, we obtain a reaction barrier of $c a .12 \mathrm{kcal} \mathrm{mol}^{-1}$ leading to an energetically degenerate state at $c a .-10 \mathrm{kcal} \mathrm{mol}^{-1}$. Proton transfer from the enol-tautomer to the nearby Asp175 increases the nucleophilic character of the enol, hence favouring the ring formation that takes place semiconcertedly. The cyclisation reaction succeeds via a BürgiDunitz angle in which the carbon atom in $\alpha$-position (C16) is perfectly oriented to form a covalent bond with the carbonyl group (C3) of the acyl-AntI complex leading to INT3. Due to 
multiple bond formations and cleavage processes, the exact barrier for the INT2 $\rightarrow$ INT3 reaction could not be determined, but the entire trajectory is energetically strongly exergonic by more than $40 \mathrm{kcal} \mathrm{mol}^{-1}$. Additionally, we find that the process is favoured by re-protonation of the Ser245 nucleophile via His355, which regenerates the active site residue. Taken together, our sampled configurations and putative DFT models manifest that release of an acetyl-AntF induce shortening of the octaketide backbone and as a consequence, forms the third aromatic ring in AQ-256.

\section{Conclusion}

Anthraquinone derivatives may contribute to the toxicity of Photorhabdus extracts against other organisms such as insects, fungi and bacteria. In most strains of Photorhabdus ${ }^{\mathbf{1 8 , 1 9}}$ corresponding gene clusters have been identified (ESI Fig. 8†), implying an important function of these natural products from this genus. In such pathways, the lyase AntI is exceptional because it shortens polyketides. The reaction mechanism of the lyase provides a unique route to the formation of the tricyclic aromatic ring (Fig. 5a) - a structural feature typically introduced by dedicated cyclases in type II PKS pathways. ${ }^{\mathbf{1 , 2 0}}$ Interestingly, AntI and its homologues are also found in type II PKS-encoding biosynthetic gene clusters of cyanobacteria and other Gramnegative bacteria (ESI Fig. $8 \dagger$ ), indicating that polycyclic aromatic polyketides are more abundant than previously thought. Furthermore, the amino acids around the proposed active centre of Ayg1p from Aspergillus fumigatus ${ }^{\mathbf{1 0}}$ and WdYg1p from Wanganella (Exophiala) dermatitidis ${ }^{\mathbf{1 1}}$ are identical to AntI (ESI Fig. 9 and 10†), and therefore they can be clearly assigned as lyases. Intriguingly, these two enzymes are produced in pathogenic fungi and play a key role in the biosynthesis of the virulence factor 1,8-dihydroxynaphthalene-melanin. Thus, the structural and functional characterisation of AntI described here opens up a hitherto unexplored mechanism for an exciting class of catalysts.

\section{Conflicts of interest}

There are no conflicts to declare.

\section{Acknowledgements}

The authors are grateful to Dr Sebastian Fuchs and Dr Darko Kresovic for MALDI-MS measurements and discussion. The Tsai and Brady labs are acknowledged for polyketide standards and the Ichinose lab for providing original data about $S$-DNPA. We thank the staff of the beamline X06SA at the Paul Scherrer Institute, SLS, Villigen (Switzerland) for assistance during data collection. This work was supported by the DFG within the SPP 1617 (H. ·B. B.), SFB 749 (M. G.), SFB 1035 (V. R. I. 'K.) and the LOEWE program of the state of Hesse as part of the MegaSyn research cluster $($ H. $\cdot$ B. $\cdot$ B. $)$.

\section{References}

1 C. Hertweck, The Biosynthetic Logic of Polyketide Diversity, Angew. Chem., Int. Ed., 2009, 48, 4688-4716.

2 J. Staunton and K. J. Weissman, Polyketide biosynthesis: a millennium review, Nat. Prod. Rep., 2001, 18, 380-416.

3 C. Khosla, D. Herschlag, D. E. Cane and C. T. Walsh, Assembly line polyketide synthases: mechanistic insights and unsolved problems, Biochemistry, 2014, 53, 28752883.

4 M. B. Austin and J. P. Noel, The chalcone synthase superfamily of type III polyketide synthases, Nat. Prod. Rep., 2003, 20, 79-110.

5 C. Hertweck, A. Luzhetskyy, Y. Rebets and A. Bechthold, Type II polyketide synthases: gaining a deeper insight into enzymatic teamwork, Nat. Prod. Rep., 2007, 24, 162.

6 Z. Zhang, H.-X. Pan and G.-L. Tang, New insights into bacterial type II polyketide biosynthesis, F1000Research, 2017, 6, 172-212.

7 A. O. Brachmann, et al., A Type II Polyketide Synthase is Responsible for Anthraquinone Biosynthesis in Photorhabdus luminescens, ChemBioChem, 2007, 8, 17211728.

8 A. Sandmann, et al., A Type II polyketide synthase from the Gram-negative Bacterium Stigmatella aurantiaca is involved in Aurachin alkaloid biosynthesis, Angew. Chem., Int. Ed., 2007, 46, 2712-2716.

9 F. Panter, D. Krug, S. Baumann and R. Müller, Selfresistance guided genome mining uncovers new topoisomerase inhibitors from myxobacteria, Chem. Sci., 2018, 9, 4898-4908.

10 I. Fujii, et al., Hydrolytic polyketide shortening by ayg1p, a novel enzyme involved in fungal melanin biosynthesis, $J$. Biol. Chem., 2004, 279, 44613-44620.

11 M. H. Wheeler, et al., New Biosynthetic Step in the Melanin Pathway of Wangiella (Exophiala) dermatitidis: Evidence for 2-Acetyl-1,3,6,8-Tetrahydroxynaphthalene as a Novel Precursor, Eukaryotic Cell, 2008, 7, 1699-1711.

12 J. D. Bauer, R. W. King and S. F. Brady, Utahmycins A and B, azaquinones produced by an environmental DNA clone, $J$. Nat. Prod., 2010, 73, 976-979.

13 T. Itoh, et al., Actinorhodin biosynthesis: structural requirements for post-PKS tailoring intermediates revealed by functional analysis of ActVI-ORF1 reductase, Biochemistry, 2007, 46, 8181-8188.

14 D. L. Ollis, et al., The $\alpha / \beta$ hydrolase fold, Protein Eng., Des. Sel., 1992, 5, 197-211.

15 L. Holm and L. M. Laakso, Dali server update, Nucleic Acids Res., 2016, 44, W351-W355.

16 C. Schleberger, P. Sachelaru, R. Brandsch and G. E. Schulz, Structure and Action of a CC Bond Cleaving $\alpha / \beta$-Hydrolase Involved in Nicotine Degradation, J. Mol. Biol., 2007, 367, 409-418.

17 M. Engel, et al., Rigidity and Flexibility of Dipeptidyl Peptidase IV: Crystal Structures of and Docking Experiments with DPIV, J. Mol. Biol., 2006, 355, 768-783. 
18 N. J. Tobias, et al., Natural product diversity associated with the nematode symbionts Photorhabdus and Xenorhabdus, Nat. Microbiol., 2017, 2, 1676-1685.

19 N. J. Tobias, et al., Genome comparisons provide insights into the role of secondary metabolites in the pathogenic phase of the Photorhabdus life cycle, BMC Genomics, 2016, 17, 537.

20 T. Taguchi, et al., Bifunctionality of ActIV as a CyclaseThioesterase Revealed by in Vitro Reconstitution of Actinorhodin Biosynthesis in Streptomyces coelicolor A3(2), ChemBioChem, 2017, 18, 316-323. 